\title{
TI.98.1
}

\section{InCommon Service Provider Onboarding - Final Report}

- PDF: InCommon-SP-Onboarding-FinalReport.pdf

- Text: InCommon-SP-Onboarding-FinalReport.txt

More Information

\begin{tabular}{|c|c|}
\hline Repository ID & TI.98.1 \\
\hline Persistent URL & http://doi.org/10.26869/TI.98.1 \\
\hline Title & InCommon Service Provider Onboarding - Final Report \\
\hline Authors & $\begin{array}{l}\text { - InCommon Streamlining SP Onboarding Working Group, including } \\
\text { Garrett King, Carnegie Mellon, https://orcid.org/0000-0002-0504-5561 } \\
\text { Tommy Roberson, Baylor, https://orcid.org/0000-0002-4722-1068 } \\
\text { Alan Buxey, MyUNiDAYS Ltd., https://orcid.org/0000-0001-8217-8379 } \\
\text { Kevin Foote, UC Boulder https://orcid.org/0000-0002-9101-1172 } \\
\text { Ken Gray, University of Michigan }\end{array}$ \\
\hline Sponsor & InCommon TAC \\
\hline Review & https://spaces.at.internet2.edu/x/rwOMBw \\
\hline Status & Preserve \\
\hline Publish Date & Sept 2018 \\
\hline DOI & $10.26869 /$ TI.98.1 \\
\hline \multicolumn{2}{|l|}{ Signature } \\
\hline Deprecated & No \\
\hline Future Review & NA \\
\hline Supersedes & NA \\
\hline Format & PDF, Text \\
\hline \multicolumn{2}{|l|}{ Related Docs } \\
\hline Development Location & https://docs.google.com/document/d/1QKs--0D_VX1_8aF77SBhbhbFznF3GAi8M5UZg0DAkzU/edit\# \\
\hline IP Framework & CC BY 4.0 \\
\hline Subject Tags & incommon federation \\
\hline Notes & \\
\hline
\end{tabular}

